\title{
PERIODONTAL LIGAMENT CELLS AS ALTERNATIVE SOURCE FOR CELL-BASED THERAPY OF TENDON INJURIES: IN VIVO STUDY OF FULL-SIZE ACHILLES TENDON DEFECT IN A RAT MODEL
}

\author{
C-F. Hsieh ${ }^{1}$, P. Alberton ${ }^{1}$, E. Loffredo-Verde' ${ }^{1}$, E. Volkmer' ${ }^{1}$, M. Pietschmann², P.E. Müller ${ }^{2}$, M. Schieker ${ }^{1}$ \\ and D. Docheva ${ }^{1,3 *}$
}
${ }^{1}$ Experimental Surgery and Regenerative Medicine, Department of Surgery, Ludwig-Maximilians-University (LMU), Nussbaumstr. 20, 80336 Munich, Germany
${ }^{2}$ Department of Orthopaedic Surgery, Physical Medicine and Rehabilitation, University Hospital of Munich (LMU), Campus Grosshadern, Marchioninistr. 15, 81377, Munich, Germany
${ }^{3}$ Experimental Trauma Surgery, Department of Trauma Surgery, University Regensburg Medical Centre, Franz-Josef-Strauss-Allee 11, 93053 Regensburg, Germany

\begin{abstract}
Tendon's natural healing potential is extremely low and inefficient, with significant dysfunction and disability due to hypocellularity and hypovascularity of tendon tissues. The application of stem cells can aid in significantly enhanced repair of tendon rupture; therefore, the main aim of this study is to assess the potential of using periodontal ligament cells (PDL), usually obtained from patients undergoing orthodontic treatment, as a novel cell source for cell-based therapy for tendon injuries in a clinically relevant rat full-size Achilles tendon defect. In addition, the study compares the differences between the healing effects of Achilles tendon-derived cells (AT) versus PDL and, hence, comprises of four experimental groups, native tendon (NT), empty defect (ED), PDL and human AT (hAT). The tendon healing in each group was assessed in the late remodelling phase at 16 weeks after surgery using a combination of methods, including evaluation of gross morphological appearance; various histological and immunohistological stainings; and detailed analyses of cell morphometry. Based on these outcome measures, PDL cell-implanted tendons exhibited not only advanced tissue maturation, less ectopic fibrocartilage formation, more organised collagen fibres, tendon matrix expression corresponding to the final healing stage, and better cellmorphometry parameters when compared with the ED group, but were also very similar to the tendons treated with hAT-derived cells. Taken together, our study clearly demonstrates the feasibility of using PDL cells as a novel cell source for tendon repair and strongly recommends this cell type for the future development of innovative regenerative applications for treatment of different tendon or ligament pathologies.
\end{abstract}

Keywords: Periodontal ligament, Achilles tendon, cellbased therapy, tendon repair, in vivo, immunohistochemistry, cell morphometry.

\footnotetext{
*Address for correspondence:

Prof. Dr Denitsa Docheva

Director of Laboratory of Experimental Trauma Surgery

Department of Trauma Surgery

University Regensburg Medical Centre

Franz-Josef-Strauss-Allee 11

93053 Regensburg, Germany
}

\author{
Telephone: +499419431605 \\ Fax: +499419431631 \\ E-mail: denitsa.docheva@ukr.de
}

\section{Introduction}

Tendons possess the capability to heal injuries in a process controlled primarily by local tendon cells, invading blood-derived cells and inducing expression changes in the surrounding extracellular matrix. Immediately after tendon injury, a scar formation is initiated, containing three overlapping stages: (1) tissue inflammation, (2) cell proliferation and (3) tissue remodelling (Docheva et al., 2015). The final remodelling stage is time consuming and is characterised by a decrease in cellularity/density, significant cell shape flattening and almost perfect cell alignment to the axis of mechanical stretch, which is accompanied by maturation of collagen type I and elastic fibrils. Additional features of this stage are a decrease in biglycan and an increase in decorin and lumican protein expression (Juneja and Veillette, 2013). In parallel, collagen and elastic fibres start to organise along the longitudinal axis of the tendon, thereby restoring tendon stiffness and tensile strength (Sharma and Maffulli, 2005a).

At present the options to heal injured tendons mainly consist of surgical intervention, tendon/ligament transfer or implantation of synthetic materials; however, they frequently result in unsatisfactory outcomes (Simonson et al., 2016). Hence, repair of tendon defects due to trauma or tendon degeneration remains as a great challenge to orthopaedic medicine. A promising new development of regenerative medicine is cell-based therapy, in which mostly stem cells are directly implanted or used for manufacturing of organoids - mimicking the original state of injured/diseased tissue. Such approach has been experimentally explored also for therapy of tendon injuries (Docheva et al., 2015). Scientists have mainly focused on two favourable cell types, namely bone morrow mesenchymal stem cells (BM-MSCs) and adipose-derived mesenchymal stem cells (AD-MSCs), because they are the best characterised and also fairly simple to obtain and expand in vitro. Interestingly, the most of the preclinical animal studies concluded that MSC delivery can lead to increased cell proliferation, but these cells often differentiated towards osteoblasts or adipocytes within the tendon area, suggesting their inherent preference to commit 
in the original lineage of the tissue from which they were isolated (Docheva et al., 2015). Subsequently, the native to the tendon-tenocytes, tendon stem/progenitor cells or tendon-derived fibroblasts became of great interest and their isolation has been successfully carried out (Bi et al., 2007). However, the use of such autologous tendon-derived cells faces several concerns. For example, the patient has to undergo additional surgery to obtain a tendon biopsy for the purification of tendon cells causing comorbidity. Another concern is if the cells are to be isolated from the injured tendon they might be compromised in terms of quality and function (Docheva et al., 2015; Sharma and Maffulli, 2005b). Furthermore, the expansion of the tendon cells to sufficient numbers for implantation will require several weeks, in which the patient will be on hold or has to revisit the hospital numerous times.

In the last years it has been recognised that MSCs exist in a wide range of tissue types, including dental and ligament tissues (Hakki et al., 2015; Lei et al., 2014; Liu et al., 2015). Periodontal ligament (PDL)-derived cells are of particular importance as in comparison to tendon cells, in a way that they are much more easily accessible through the oral cavity and can be simply harvested especially from extracted teeth when patients have orthodontic treatments (Miura et al., 2003; Seo et al., 2004). Recently, several groups, including our, reported that PDL contains endogenous stem/progenitor cells (Bluteau et al., 2008; Docheva et al., 2010; Ivanovski et al., 2006), which express tendon-specific genes such as scleraxis and tenomodulin (Docheva et al., 2010).

The above findings strongly motivated us to investigate if PDL-derived cells can be used for cell-based therapy of tendon injuries. PDL cells hold several advantages to tendon-derived cells in regenerative applications, namely: easier obtaining and banking; avoiding additional surgery with potential risk for pain and infection and decreasing donor comorbidity. For this we designed not only an in vivo testing of the potential of PDL cells to form tendonlike structures, but actually directly implanted the cells in a clinically relevant tendon injury model by utilising our established surgically created full-size tendon defects in rat Achilles tendons. In order to estimate the regenerative capacity of the PDL cells we compared the tendons receiving PDL cells to tendons implanted with Achilles tendon (AT)-derived cells as well as to non-implanted empty defect tendons. We assessed, by evaluation of gross morphological appearance, various histological and immunohistological stainings, and detailed cell morphometric analyses, how advanced is the healing process at 16 weeks after surgery and implantation, a time point corresponding to the late tendon repair phase. The results presented below strongly demonstrated that PDL cells contributed to tendon repair, which was very comparable to that of AT cells and far more superior to non-implanted tendons, hence concluding their feasibility as alternative cell source holding many advantages for therapy of tendon injuries.

\section{Materials and Methods}

\section{Cell preparation prior implantation}

In the study two standard cell lines characterised before, namely PDL-(hTERT) cell line from Docheva et al. (2010) and human AT-(hTERT) cell line derivative from Kohler $e t$ al. (2013) were implemented. Both cell lines were cultured in DMEM/Ham's F-12 (1:1 mixture) medium supplemented with stable glutamine $(365.3 \mathrm{mg} / \mathrm{L}), 1 \times$ MEM amino acids (all from Biochrom, Berlin, Germany), $10 \%$ FBS and $1 \%$ L-ascorbic acid-2-phosphate (both from SigmaAldrich, Steinheim, Germany) in a humidified incubator at constant $37^{\circ} \mathrm{C}$ and $5 \% \mathrm{CO}_{2}$. Prior to implantation, the cells were expanded in three consecutive passages to $12 \times 10^{6}$ corresponding to $1 \times 10^{6}$ cells per animal.

\section{Animal model and surgical procedure}

The surgical procedures were carried out according to our established in vivo model (Pietschmann et al., 2013). The procedures including animal handling prior, during and after surgery were approved by the Animal Care and Use Committee of the Bavarian Government (Animal Grant Nr. 55.2-1-54-2531-58-08). Thirty athymic female Foxn1nu rats were purchased from Charles River Laboratories (Sulzfeld, Germany). Four different study groups were randomly selected (with ten animals per group): (1) native tendon (NT), non-injured left Achilles tendon; (2) empty defect (ED) control group, consisting of injured right Achilles tendon without cell implantation; (3) and (4) in which the animals received one-shot implantation of PDL(PDL group) or hAT-cell pellets (hAT group) within injured right Achilles tendon, respectively. In the experimental design, in order to reduce numbers of animals sacrificed in the study, injury was applied to the right leg, while the left, non-injured was used as native tendon control.

In brief, the surgical procedure consisted of: (1) animal anaesthesia was carried out; (2) the right Achilles tendon was exposed with a straight skin incision; (3) three millimetres of tissue from the Achilles tendon proper were resected by a surgical removal; (4) the defect was reconnected and stabilised with monofilament PDS (polydioxanone) 5-0 suture material by Kirchmayr-Kessler suture; (5) the original gap of $3 \mathrm{~mm}$ was kept and no end-toend was done in order not to shorten the tendon; (6) a three dimensional cell pellet of $1 \times 10^{6}$ cells was administrated into the defect site with a sterile pipette tip; and (7) the skin was sutured. After surgery the rats were allowed to walk freely in their cages and evaluated for tendon repair after 16 weeks. For this all animals were sacrificed by euthanasia and gastrocnemius muscle-Achilles tendon-calcaneus bone units were retrieved after skin incision, imaged and total length of the total Achilles tendons and width of tendon proper of the Achilles tendons were manually measured.

\section{Histology \\ Processing}

The explanted gastrocnemius muscle-Achilles tendoncalcaneus bone units were fixed either in $95 \%$ ethanol/ $5 \%$ absolute acetic acid overnight at $4{ }^{\circ} \mathrm{C}$, or in $4 \%$ 
paraformaldehyde (PFA) (Merck, Darmstadt, Germany) (five animals in each fixation). After fixation, specimens were decalcified in $10 \%$ EDTA/PBS pH 8.0 (SigmaAldrich, Munich, Germany) for four weeks at room temperature, then embedded in tissue freezing medium (Jung, Leica, Nussloch, Germany) and cryosectioned at $12 \mu \mathrm{m}$ (Microm HM500 OM, Fisher, Walldorf, Germany). Prior use sections were stored at $-20^{\circ} \mathrm{C}$.

\section{Section hydration prior staining, dehydration and mount-} ing after staining

All the sections before staining were equilibrated to room temperature and hydrated with PBS $3 \times 5$ min then changed to deionised water. After following staining, Haematoxylin and Eosin (H\&E), Safranin O \& Fast Green (S.O/F.G.), Periodic Acid-Schiff (PAS) and 3,3 diaminobenzidine (DAB)-based staining, routine dehydration and mounting were carried out as follows: sections were washed through a graded ethanol series for 5 min each from $70 \%, 80 \%$, $90 \%$, then $100 \%$ ethanol and xylene (Carl Roth, Karlsruhe, Germany) $2 \times 5 \mathrm{~min}$, and last mounted with Entellan mounting medium (Carl Roth, Karlsruhe, Germany).

\section{$H \& E$}

Samples were placed in Haematoxylin (ready for use solution) (Carl Roth, Karlsruhe, Germany) in distilled water for $5 \mathrm{~min}$, rinsed under deionised water for $5 \mathrm{~min}$, washed in tap water for $7 \mathrm{~min}$. Next, samples were immersed in Eosin (ready for use solution) (Carl Roth, Karlsruhe, Germany) for $5 \mathrm{~min}$, changed to distilled water for $30 \mathrm{~s}$, dehydrated and mounted.

\section{S.O/F.G. \& quantification}

Samples were placed in $0.02 \%$ Fast Green (Sigma-Aldrich, Steinheim, Germany) in $95 \%$ ethanol for $12 \mathrm{~min}$, rinsed under $1 \%$ acetic acid in distilled water for $5 \mathrm{~min}$, immersed in $0.5 \%$ Safranin O (Sigma-Aldrich, Steinheim, Germany) in water for $15 \mathrm{~min}$. Last, samples were rinsed quickly in $90 \%$ and $100 \%$ ethanol and mounted. In order to cover the entire tendon territory, approx. 50 consecutive images/ section were taken and digitally stitched with Adobe Photoshop CS5 software (Adobe System, San Jose, CA, USA). Next, total Achilles tendon area (denominator) was measured in pixel with "magic wand" tool and by using "select colour range" tool the red-to-orangepositive pixels (numerator), corresponding to sulphate glycosaminoglycans (GAGs) areas, were automatically quantified. Final data were expressed as a percentage of GAGs and for each study group mean values and standard deviations were calculated.

\section{$P A S$}

The PAS staining kit was obtained from Sigma-Aldrich. Samples were placed in periodic acid solution for $5 \mathrm{~min}$, rinsed 3 times under distilled water, immersed in Schiff's reagent (St. Louis, MO, USA) for $15 \mathrm{~min}$, washed in running tap water for $5 \mathrm{~min}$. Next, samples were stained in haematoxylin solution for $90 \mathrm{~s}$, rinsed in running tap water, dehydrated and mounted.

All stainings were reproduced thrice independently as three to five animals/group were analysed.

\section{Immunohistochemistry}

Antibodies

Primary: biglycan (ab58562, 1:30 dilution), collagen I (ab34710, 1:100), collagen III (ab7778, 1:50) and elastin (ab21610, 1:30, all from Abcam, Cambridge, UK); decorin (sc-22753, 1:30) and lumican (sc-33785, 1:50) (both from Santa Cruz, Heidelberg, Germany); osteopontin (MPIIIB101, 1:20, DSHB, Iowa City, IA, USA); (HLA)-ABC (M0736, 1:30, Dako, Glostrup, Denmark). Secondary: anti-goat Cy3 (705-165-147, 1:500), anti-rabbit FITC (111-095-144, 1:500), anti-mouse Cy3 (115-165-146, 1:500) (all from Jacksonimmuno, West Grove, PA, USA) and anti-mouse vectastain ABC kit (PK-4002, 1:250, VectorLab, Peterborough, UK).

\section{Immunolabelling procedure}

Selected sections were treated with $2 \mathrm{mg} / \mathrm{mL}$ hyaluronidase (Sigma-Aldrich, Steinheim, Germany) for $30 \mathrm{~min}$ at $37{ }^{\circ} \mathrm{C}$. After washing and blocking, either with $2 \%$ skimmed milk (Sigma-Aldrich, Steinheim, Germany) / PBS or $2 \%$ BSA (Sigma-Aldrich, Munich, Germany) / PBS, primary antibodies were applied overnight at $4{ }^{\circ} \mathrm{C}$. Next day, corresponding secondary antibodies were used for $1 \mathrm{~h}$. For immunofluorescence-based experiments, sections were shortly counterstained with 4',6-diamidino2-phenylindole (DAPI) (Life technology, Carlsbad, CA, USA) and mounted with Fluoroshield (Sigma-Aldrich, Munich, Germany). For DAB-based experiments, an initial quenching step was performed using $4 \% \mathrm{H}_{2} \mathrm{O}_{2} /$ methanol for $30 \mathrm{~min}$ at room temperature, and afterwards the classical vectastain $\mathrm{ABC}$ kit was used according to the manufacturer's instructions (VectorLab, Eching, Germany), then sections were dehydrated and mounted.

All stainings were reproduced thrice independently with three to five animals/group analysed.

\section{ELISA quantification of collagen type I, biglycan and lumican protein expression}

PFA-fixed sections mounted on microscope slides (90 sections each animal, 5 animals per group) were used for protein extraction. Briefly, only Achilles tendon area was collected manually by scalpel. The tissue material was lysed in extraction buffer, composed of $8 \mathrm{M}$ urea (Merck, Hohenbrunn, Germany), 50 mM Tris-HCl (Merck, Darmstadt, Germany), 1 mM EDTA (Carl Roth, Karlsruhe, Germany), 1 mM DTT (BIO-RAD, Munich, Germany), by incubation at $4{ }^{\circ} \mathrm{C}$ for $30 \mathrm{~min}$, followed by centrifuged for $20 \mathrm{~min}$ at $1 \times 10^{4} \times \mathrm{g}$, and supernatants containing protein extracts were stored at $-20{ }^{\circ} \mathrm{C}$ until further use. Protein concentration was determined using the Micro BCA Protein Assay Kit (Thermo, Rockford, IL, USA) according to the manufacturer's protocol. The protein samples were normalised between animals and further diluted 1:50 in order to lower the antigen concentration to the detection range of the ELISA kits. Commercially available ELISA kits for collagen type I (LS-F4691) biglycan (LS-F15938, both from LifeSpan BioScience, Seattle, WA, USA) and lumican (CSB-EL013234RA, Cusabio Biotech, College Park, MD, USA) were used following the manufacturer's instructions and twice independently reproduced with 5 animals per group. 


\section{Quantification of collagen type III}

An automated quantitative image analysis was performed as described in McCloy et al. (2014) with slight modifications. In brief, using Image J (National Institutes of Health, Bethesda, MD, USA) the following algorithm was applied: (1) nine randomly $10 \times$ magnification images were taken within the area of injury; (2) area of interest was manually designated using the "drawing/selection" tool; (3) "set measurements" for area, integrated density and mean grey value was selected from the analyse menu; (4) last the corrected total cryosections fluorescence (CTCF) representing the collagen III expression was exported and calculated in Excel (Microsoft) as follows CTCF $=$ media of integrated density - media of the selected area $\times$ mean fluorescence. Three animals per group (each with nine images) were analysed.

\section{Elastic fibres scoring}

A point-based scoring system for elastic fibres assessment was used according to Hsieh et al. (2016) and legend of Table 2. Elastin-stained sections from three animals (each animal represented with 3 to 5 different slides consisting of 3 to 4 sections) were employed for microscopical scoring in a blinded fashion.

\section{Classical microscopy}

Photomicrographs of H\&E, S.O/F.G., PAS, DAB-based stainings were taken with a colour AxiocamICc3 camera, while images of immunohistochemical stainings were taken with a black/white Axiocam MRm camera, both mounted on Observer Z1 microscope (Carl Zeiss, Jena, Germany).

\section{Polarised microscopy $\&$ evaluation}

H\&E-stained sections from the 4 study groups ( 3 animals per group and each animal represented with 3 different sections) were imaged using $10 \times$ objective supplemented with polarised filter mounted on Axioskope 2 microscope (Carl Zeiss, Jena, Germany). Under this filter, organised collagen type I fibres can be visualised in orange to yellow colours when exposed to polarised light. For final representation, consecutive images of the whole unit of gastrocnemius muscle-Achilles tendon-calcaneus bone were digitally stitched together with Adobe Photoshop CS5 software (Adobe System).

Organised collagen type I fibres have strong birefringence property when exposed to polarised light; hence, they can be visualised in orange to yellow colours using microscopy. Microscopy was performed with $10 \times$ objective supplemented with a polarised filter mounted on Axioskope 2 microscope. For optimal imaging the transmission axis of the analyser was with an angle of $54^{\circ}$ to the axis of the polariser in the analyses of all samples. In order to cover the entire tendon territory, approx. 50 consecutive images/section were taken manually in a mosaic manner and digitally stitched with Adobe Photoshop CS5 software (Adobe System). Next, total Achilles tendon area (denominator) was measured in pixel with "magic wand" tool and by using "colour selecting" tool the yellow-to-orange-positive pixels (numerator), corresponding to highly organised collagen areas, were automatically quantified. Final data were expressed as a percentage of collagen birefringence and for each study group mean values and standard deviations were calculated.

\section{Quantification of nuclear aspect ratio and density}

Determination of cell density by nuclear count and aspect ratio of nuclei (NAR, defined as the ratio of the minor diameter to the maximal diameter) was performed with the following algorithm: (1) 9 randomly taken $20 \times$ magnification DAPI-stained images within the area of injury, $3 \mathrm{~mm}$ proximal from the calcaneus, for 3 to 5 animals per group were taken; (2) DAPI images were analysed using ImageJ software v1.49 with installed NII plugin (National Institutes of Health, Bethesda, MD, USA); (3) each image was converted to greyscale by colour threshold adjustment (DAPI-stained areas in black and background in white); (4) the total number of nuclei/image and the minimal and maximal diameter for each nucleus were automatically measured by "analyse particles" tool in NII plugin; (5) the obtained values were exported to an Excel spreadsheet (Microsoft); (6) average cell density per group was expressed by calculating the mean number of nuclei per $\mathrm{mm}^{2}$; (7) NAR was calculated in Excel and distributed by frequency using GraphPad Prism 5 software (GraphPad, La Jolla, CA,USA). Rounded cells are represented by NAR $=1$, while flattened cells have NA $=0$ and all nuclei/group were assessed calculation of NAR.

\section{Quantification of nuclear orientation}

To determine nuclear orientation by estimating angular deviation, H\&E-stained images were analysed using the AxioVision V4.8 software (Carl Zeiss, Jena, Germany). Six randomly taken $20 \times$ magnification images for 3 to 5 animals per group were analysed and a total number of approx. 200 nuclei/group was evaluated for angular deviation. The angle measurements between the longitudinal axis of the tissue and the major axis of a nucleus were automatically given with the "angular" tool for manually selected nuclei. The angular deviation was distributed by frequency with GraphPad Prism 5 software (GraphPad). Nuclear orientation angle with values of $0^{\circ}$ represents a nucleus that is perfectly aligned along the longitudinal axis and as this value increases when the nucleus becomes more angled to the long axis of the tendon.

\section{X-ray analysis}

In-tendinous calcification was screened in dissected hind limbs from the four study groups (ten animals/group) using $\mathrm{X}$-ray imaging with a Cabinet X-ray Faxitron $(32 \mathrm{kV}, 2 \mathrm{~mA}$ and $2 \mathrm{~s}$ exposure time, Hewlett Packard, McMinnville, OR, USA). The total area of calcification in each animal was digitally quantified from X-ray images using the "polygonal" tool of Image Pro Plus software v4 (Media Cybernetics, San Diego, CA, USA). Mean value and standard deviations were calculated for each study group (ten animals/per group) with GraphPad Prism 5 software (GraphPad). 


\section{Data and statistics analysis}

Quantitative data and statistical significance analyses were done with GraphPad Prism 5 software (GraphPad). Bar charts show mean values and standard deviations. Multi group statistical testing was performed with one-way ANOVA followed by post-hoc Dunnett's test and a $p$-value of $<0.05$ was considered statistically significant.

\section{Results}

\section{Gross and morphological appearance of Achilles tendons after cell implantation}

After 16 weeks of surgery the gross appearance and morphological organisation of the Achilles tendons were assessed by imaging, length and width measurements and H\&E stainings. The tendon defect ends were re-connected in all injured groups, including the ED group. However, the Achilles tendons of the PDL and hAT groups had more opalescent tissue appearance in comparison to the ED group (Fig. 1a). Furthermore, according to our quantitative data, these two groups exhibited smaller mean tendon width compared to the ED group, whereas no significant differences were found regarding the mean tendon length (Table 1). H\&E-stained mosaic imagining of the four experimental groups were performed (Fig. 1b) and used for examination of the overall morphological organisation and composition of different tissues sub-areas such as tendinous regions (from the NT group), areas of high cellularity, ectopic calcification, and adipose deposition (from the ED group) (Fig. 1c). In the control native Achilles tendons (Fig. 1b, NT), well organised parallel collagen fibres are clearly visible; the tendon tissue is very homogeneous with low number of cells, while some adipose and loose connective tissue can be found on the outer side of the Achilles tendons. In contrast, in the ED group large ossified and vascularised areas surrounded with poorly organised fibrous tissues containing high cell numbers as well as occasionally fatty deposits were characteristic features (Fig. 1b, ED). The cell-implanted PDL (Fig. 1b, PDL) and hAT (Fig. 1b, hAT) groups were very similar to each other and when compared to the NT group, they were clearly larger and more heterogeneous. The Achilles tendons in these groups were mostly composed in the
Table 1. Mean length and width of the four study groups.

\begin{tabular}{|c|l|l|}
\hline Study group & Length $[\mathbf{c m}]$ & Width $[\mathbf{m m}]$ \\
\hline NT & $1.14 \pm 0.12$ & $1.60 \pm 0.23$ \\
\hline ED & $1.32 \pm 0.14$ & $3.38 \pm 0.59$ \\
\hline PDL & $1.32 \pm 0.10$ & $2.84 \pm 0.36^{*}$ \\
\hline hAT & $1.34 \pm 0.14$ & $2.65 \pm 0.49^{* *}$ \\
\hline
\end{tabular}

Values present mean \pm standard deviation; $* p=0.03$ PDL $v s$. ED and $* * p=0.0074$ hAT vs. ED.

periphery of larger tendinous areas with well-organised collagen fibres and in the centre with more fibrous regions with higher cellularity indicating still ongoing tissue remodelling. However, ectopic calcification formation appeared scarce and smaller in size, which represented a major difference between PDL and hAT groups to the ED group. This first assessment of the PDL-implanted Achilles tendons suggested that this group performs comparably to the hAT group, but also is superior to the non-implanted injured tendon group, ED. To fully validate our initial observations, we carried out detailed molecular analyses of the ectopic calcification and tendon matrix composition as well as assessment of characteristic cellular features by cell morphometry quantifications.

\section{Evaluation of ectopic calcification formation}

In order to carefully compare the in-tendinous ossification we first analysed the overall content of related to the initial fibrocartilage glycosaminoglycans and mucopolysaccharides by S.O/F.G. and PAS staining of all groups (Fig. 2a and Fig. 2b). A drop in the deposition of non-tendinous glycosaminoglycans/proteoglycans and mucopolysaccharides is an indication for advanced remodelling and entry in the maturation phase of the tendon repair process. Among the three injured groups, S.O/F.G.-rich areas and very strongly positive PAS staining were detected only in the ED group mainly around the ectopic calcification areas. This observation was further confirmed by quantification of S.O-positive area (Table 2 ). Then, by using an antibody directed against the bone

Table 2. Quantification of S.O.-positive area, calcified area and total collagen birefringence and scoring of elastic fibres.

\begin{tabular}{|c|c|c|c|c|c|}
\hline Study group & $\begin{array}{c}\text { S.O. positive area } \\
{[\%]}\end{array}$ & $\begin{array}{c}\text { Calcified area } \\
{\left[\mathbf{m m}^{\mathbf{2}}\right]}\end{array}$ & $\begin{array}{c}\text { Total collagen } \\
\text { birefringence [\%] }\end{array}$ & \multicolumn{2}{c|}{ Scoring of elastic fibres } \\
\cline { 4 - 6 } & $2.83 \pm 0.96$ & $0 \pm 0$ & $81.34 \pm 9.07$ & 4 & 4 \\
\hline NT & $15.46 \pm 5.88$ & $3.79 \pm 1.04$ & $24.15 \pm 9.53$ & 2.25 & 1.25 \\
\hline ED & $7.50 \pm 4.11$ & $2.45 \pm 0.73$ & $30.87 \pm 8.51$ & 3 & 3 \\
\hline PDL & $11.03 \pm 2.59$ & $2.98 \pm 0.90$ & $33.88 \pm 9.36$ & 3 & 3.5 \\
\hline hAT &
\end{tabular}

Quantification digital image analysis of S.O. stainings, X-ray and polarised light microscopy. Scoring was done in a blinded fashion using the following point system: for content the total amount of elastin fibres (non-mature or mature) compared to NT was estimated by 4 points $=0-25 \%, 3$ points $=26-50 \%, 2$ point $=51-75 \%$ and 1 point $=76-100 \%$, for elastic fibres morphology 4 points $=$ mature, thin parallel elastic fibres, while 1 point $=$ non-mature, thick irregular elastic fibres. 
(a)

(b) $\mathrm{H} \& \mathrm{E}$
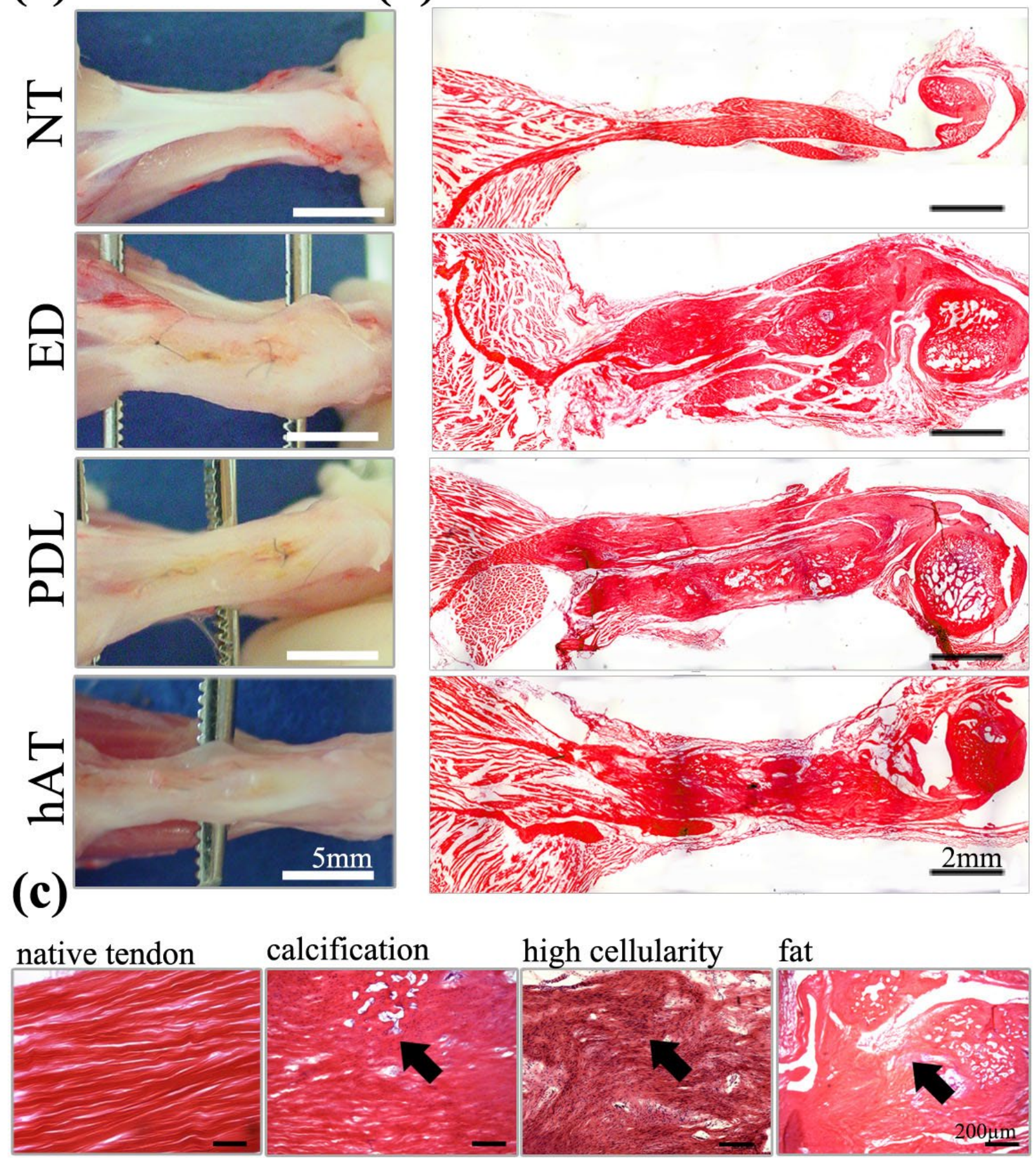

fat

Fig. 1. Overall appearance and tissue morphology of the Achilles tendons in the four study groups. (a) Representative gross morphological images and (b) representative H\&E mosaic photomicrographs of NT, ED, and PDL- and hATimplanted Achilles tendons after 16 weeks of healing. (c) Examples of H\&E micrographs demonstrating different tissue sub-areas such as tendinous areas (from NT group), areas of high cellularity, ectopic calcification, and adipose deposition (from ED group) in the Achilles tendon explants.

marker osteopontin (Fig. 2c) and by quantifying the mean area of ectopic calcification on X-ray images of 10 animals per group (Table 2) it was revealed that the dimensions of calcification in PDL and hAT group are very comparable. Due to detected in-group variability, no significant difference but a tendency was found to ED group in terms of ossification. In total, the above analyses revealed that the implantation of either PDL or hAT cells was advantageous for the tendon healing process.

\section{Expression and organisation of collagen and tendon-} related matrix proteins

We analysed the expression of the two major collagens, namely collagen I and III that are critically involved 
(a)
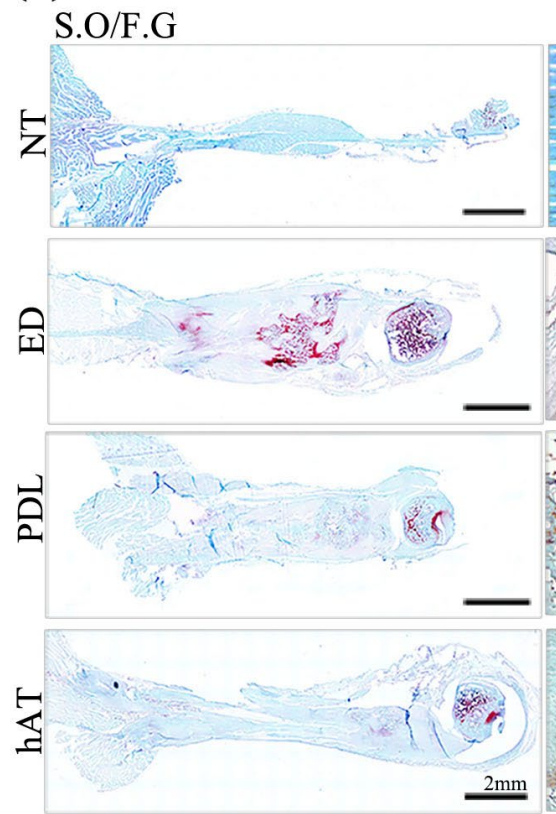

(b)

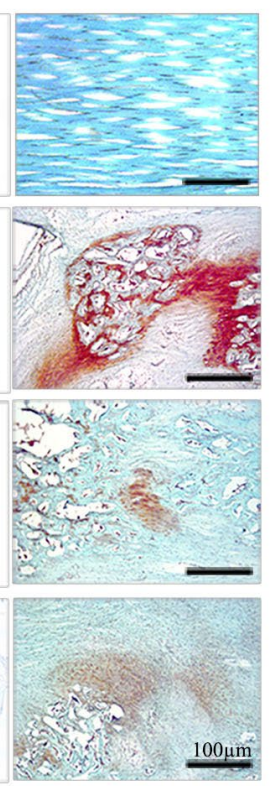

(c)

PAS
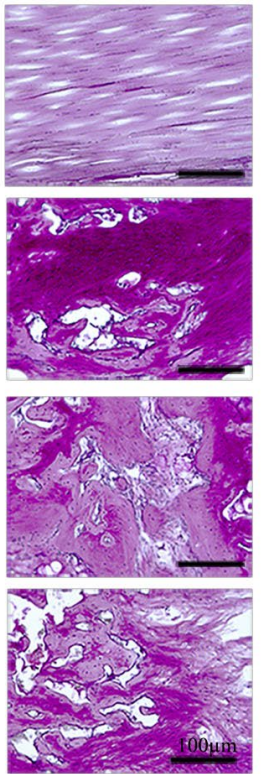

osteopontin
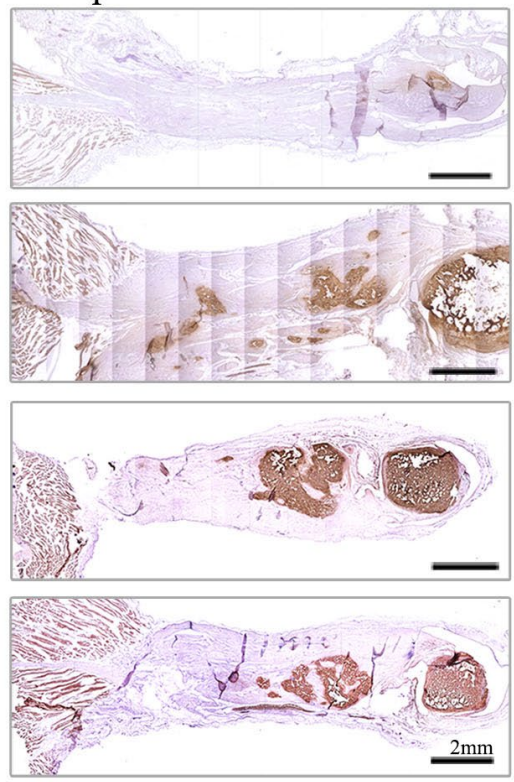

Fig. 2. Analyses of glycosaminoglycans- and mucopolysaccharides-rich areas and ectopic calcification in the Achilles tendons. (a) Representative mosaic and magnified images of Safranin O (S.O, orange to red colour)/ Fast Green (F.G., green to light blue colour), (b) Periodic Acid-Schiff (PAS, dark purple colour) and (c) bone marker protein - osteopntin stainings. All staining were reproduced thrice independently with sections from 5 animals/group.

during tendon healing. Our results showed that the PDL and hAT groups had higher immunofluorescence signals for collagen type I and lower signals for collagen type III than the ED group (Fig. 3a and b). This observation was further confirmed by polarised light microscopy (Fig. $3 \mathbf{c}$ ) and quantitative analyses (Table 2), demonstrating increased collagen birefringence, equivalent of more superior organised and mature collagen fibres, in the Achilles tendons from the PDL and hAT groups compared to the ED. To quantify collagen type I (Fig. 3d) and III (Fig. 3e) expression, we next carried out ELISA and automated digital image analysis, respectively. The quantitative evaluation demonstrated that the concentration of collagen type I was significantly higher in the hAT group followed by the PDL. With regards to collagen III expression, all injured groups had significantly higher levels than NT group. A tendency for lower collagen III expression in PDL group was detected (Fig. 3e).

Then, immunofluorescence-based studies of four different antigens - biglycan, decorin, lumican and elastin - were carried out (Fig. 4a and Table 2). In general, advanced remodelling and the late maturation phase of the tendon repair process is characterised not only by decreased ratio of collagen type III to collagen type I but also by reduction in biglycan (regulator of early and thin collagen fibril formation) and upregulation of decorin, lumican (regulators of late and thick collagen fibril formation) and elastin levels. Such molecular pattern is typical for the control non-injured NT group. The exact biglycan and lumican protein levels were measured with target-specific ELISA. The obtained quantification data not only confirm the immunofluorescence results, but also revealed a significantly lower biglycan (Fig. 4b) and significantly higher lumican (Fig. 4c) expression in PDL and hAT groups. Finally, scoring of elastin fibres contend and morphology suggested pattern more close to the NT in PDL and hAT groups.

All in all, our analyses of the selected proteins revealed in the PDL group a tissue composition very comparable to hAT group, and furthermore closer to the NT group. In contrast, ED group exhibited high levels of biglycan and low levels of decorin, lumican and elastin. Altogether, based on our investigation outcomes we concluded that the PDL group, similar to the hAT group, has more mature and organised matrix protein composition than the ED group.

\section{Cell morphometric analyses}

The above described changes of the tendon matrix in advanced stage of tendon healing are further accompanied with lowering of cell density, cell flattening and parallel alignment to the tensile axis. Therefore, we first evaluated cell density in the four experimental groups by performing nuclear counting on DAPI images and found that the cell densities of PDL- and hAT-implanted Achilles tendons are significantly lower than the ED group (Fig. 5a). Next, we assessed cellular alignment by analysing the frequency distribution of angular degrees of cells within the Achilles tendons of the four study groups. In the NT group more than $95 \%$ of the nuclei were aligned in the range of $0-20^{\circ}$ angle to the longitudinal tendon axis. In the PDL and hAT groups about $60 \%$ of the nuclei were within the angular deviation range of $0-20^{\circ}$, while in the ED more than $60 \%$ of the nuclei had angular deviation of above $30^{\circ}$ (Fig. 5b). Finally, we quantified the frequency distribution of the nuclear aspect ratio (NAR), which directly reflects the flattening of cells (Fig. 5c), and found in NT group 
(a)

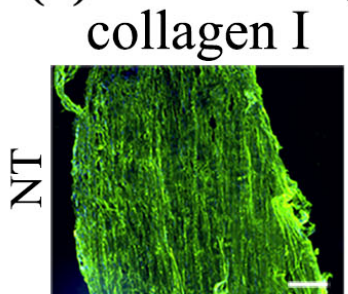

(b)
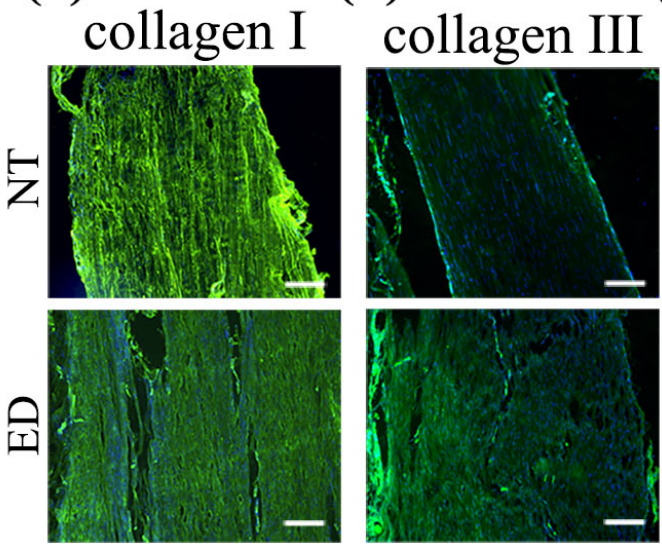

(c)
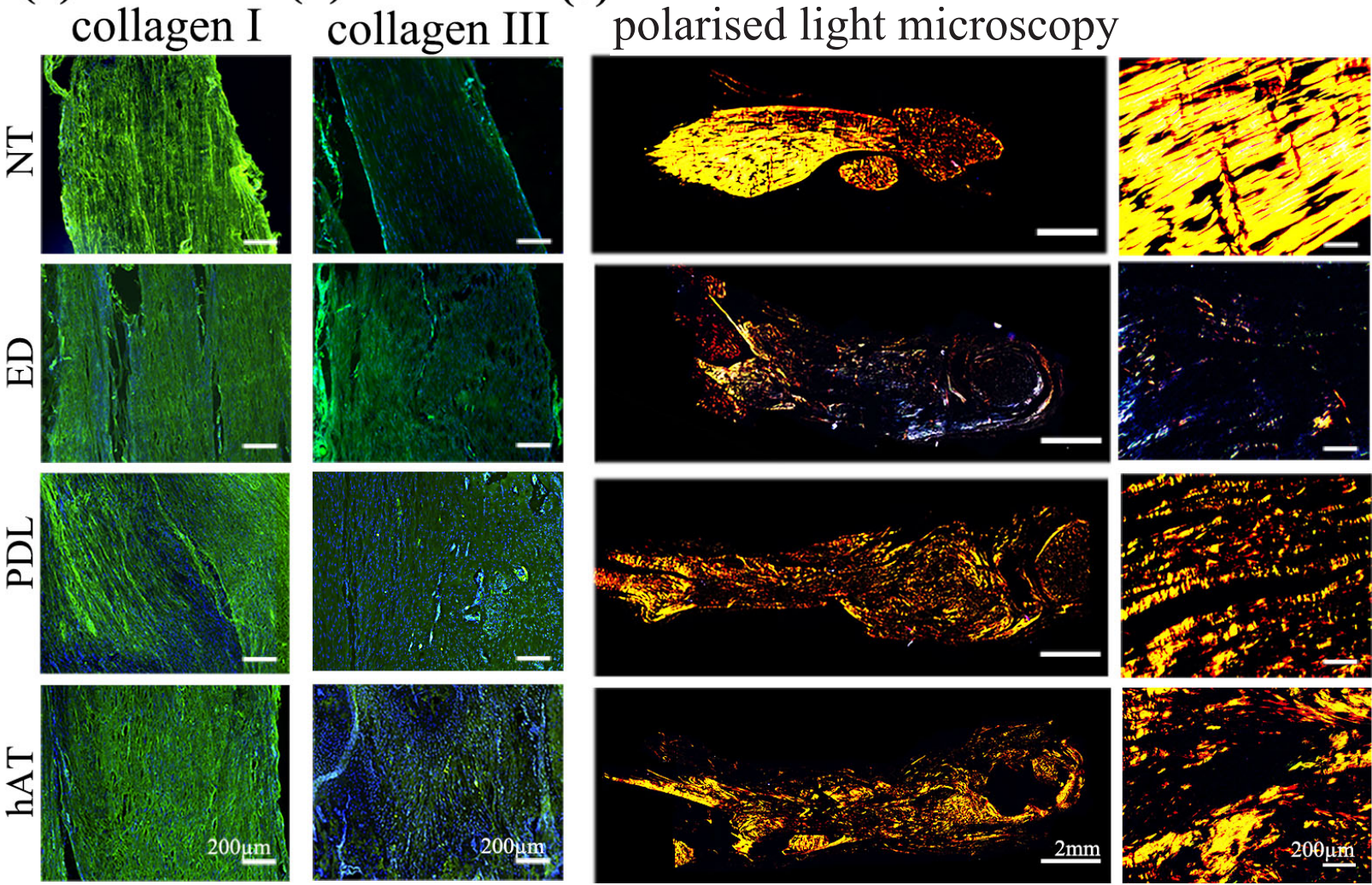

(d)

(e)

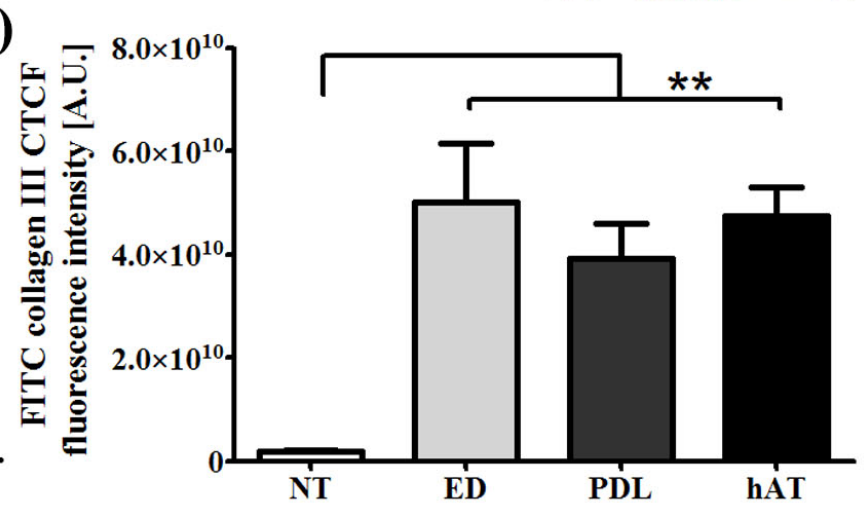

Fig. 3. Expression and organisation of collagen proteins. (a) Collagen type I and (b) Collagen type III immunofluorescent stainings (in green colour) and nuclear counterstain DAPI (in blue). (c) Representative mosaic and magnified images of H\&E-stained Achilles tendons under polarised light, wherein highly organised collagen fibres are visible in orange to yellow colours. All stainings were reproduced thrice independently, including sections from 3 to 5 animals/group. (d) Collagen type I levels were assessed by ELISA. Bar charts present mean \pm standard deviation; $* * * p<0.005$, $* * \mathrm{p}<0.01$. (e) The collagen type III protein expression was quantified by automated digital image analyses and expressed as CTCF (corrected total cryosection fluorescence) in arbitrary units (A.U.). Bar charts present mean \pm standard deviation; $* * p=0.0025$.

that the majority of the cells, approx. $85 \%$, have NAR of 0.0-0.2. Interestingly, in the PDL group the frequency of cells with $0.0-0.2$ NAR was approx. $60 \%$ and thus being higher than that of ED and even hAT groups, and moreover the frequency of cells with NAR 0.5-1.0 was very similar to that of NT group.

To examine whether the implanted PDL and hAT cells were integrated in the Achilles tendon, we have performed cell tracking by staining with anti-human HLA-ABC antibody (Fig. 5d). No signals were observed in NT and ED groups as expected, while in the PDL and hAT groups, incorporated human cells surviving or being their progeny were detected suggesting direct contribution as well as long-term survival in the defect area.

Taken together, based on the cell morphometry data together with the above-described results obtained in a very clinically relevant in vivo model, we firmly concluded that implantation of PDL cells aids in Achilles tendon healing that is on one side comparable to hAT cells and on the other more advanced and superior to the healing of ruptured non-implanted tendons. 
(a)

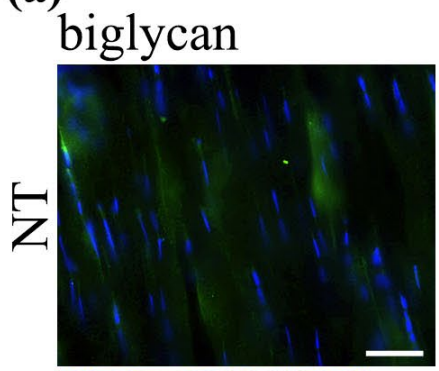

decorin
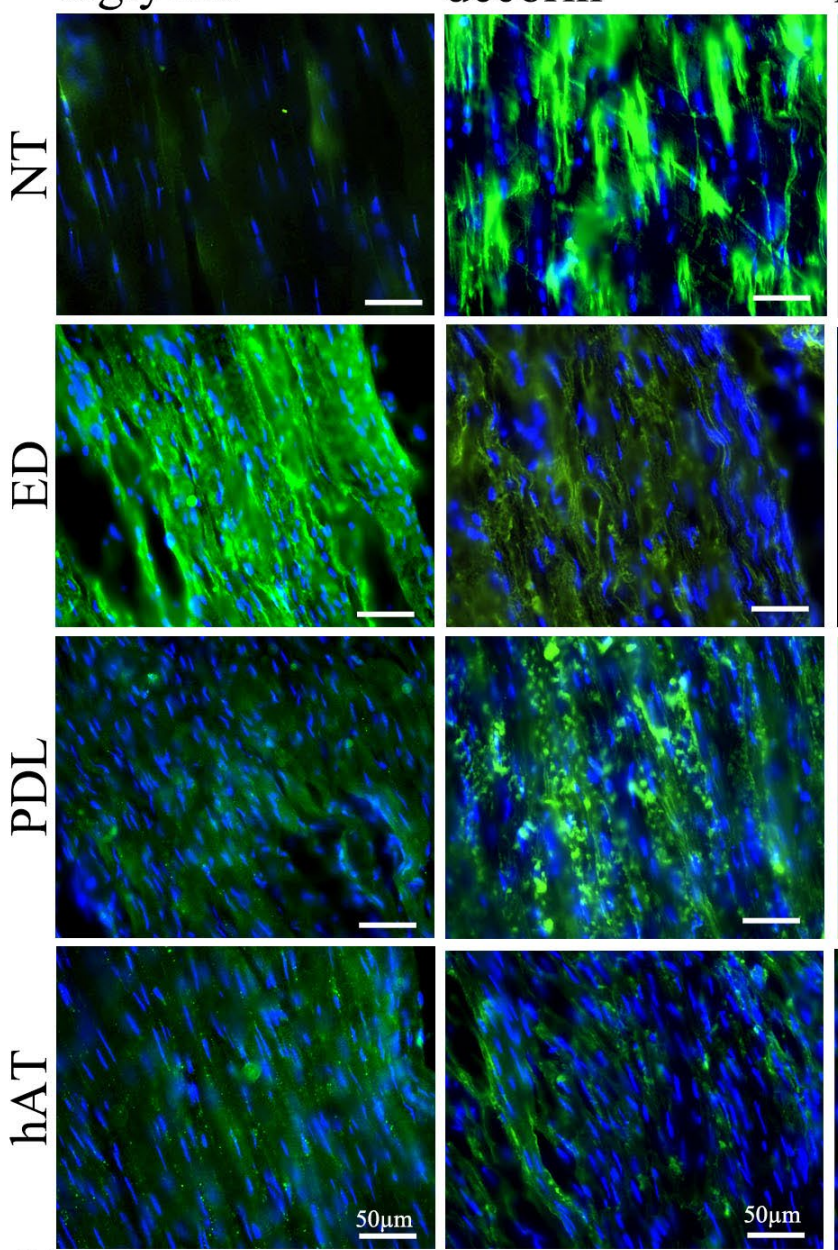

(b)
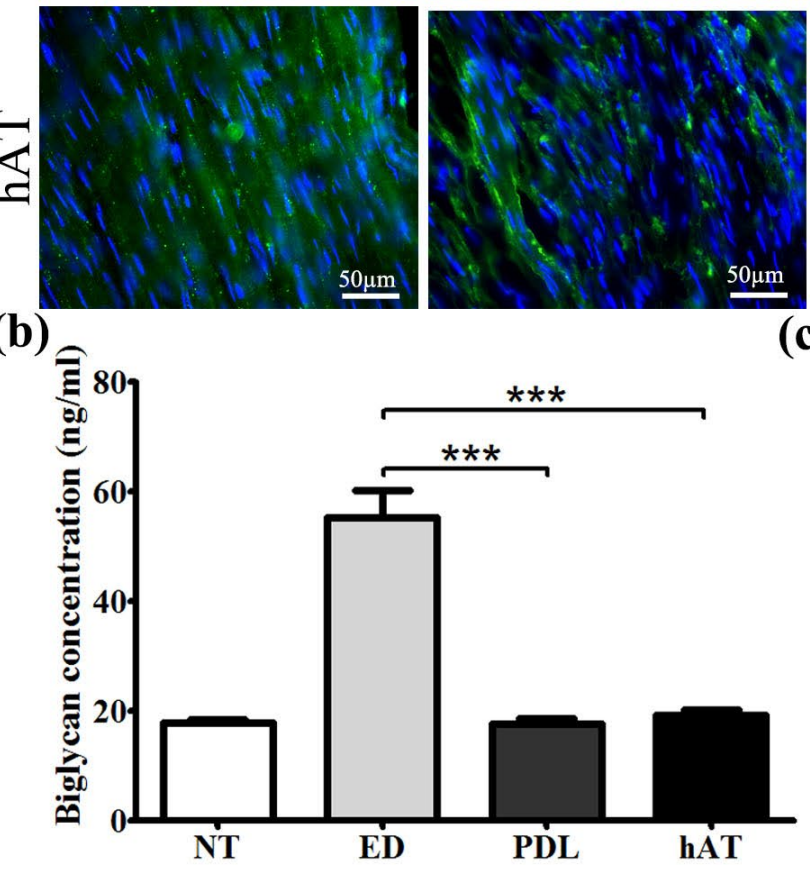

lumican
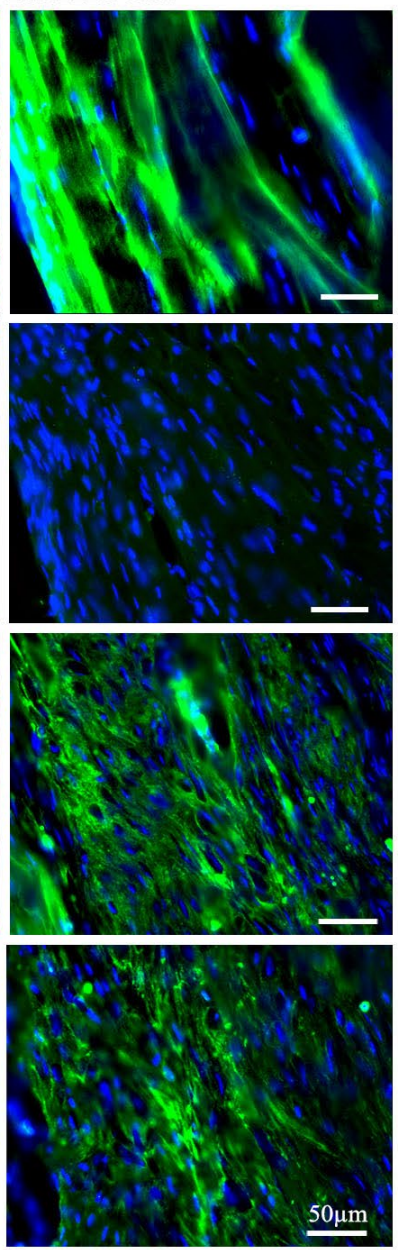

(c)

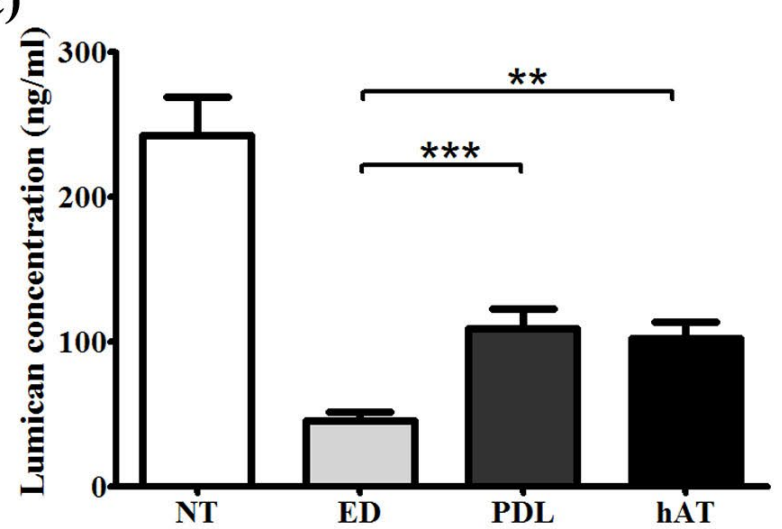

Fig. 4. Expression of key proteoglycans and elastin fibres. (a) Representative immunofluorescent images of the tendon-related matrix proteins biglycan, decorin, lumican and elastin (all in green colour) and nuclear counterstain DAPI (in blue) expressed in the Achilles tendons of the four study groups, NT, ED, PDL and hAT. All staining were reproduced thrice independently, including sections from 3 to 5 animals/group. (b) Biglycan and (c) lumican protein levels quantified by ELISA. Bar charts present mean \pm standard deviation; $* * * p<0.005, * * p<0.01$.

\section{Discussion}

The frequent failure of fully satisfying tendon repair following age-related degeneration and overuse tendon ruptures has encouraged the search for novel therapeutic approaches to enhance the healing process of tendons. A major theme in the ongoing strategies is in the application of stem cells in injured tendons (Lui and $\mathrm{Ng}$, 2013; Oragui et al., 2012; Siddiqui et al., 2010). Stem cells can be simply defined as a cellular population with the ability to selfreplicate through mitosis to form daughter cell lines, which have the potential to terminally differentiate into a number of different cell lineages depending on the signalling milieu reaching these cells. In recent years, several tissues such as bone marrow, fat or tendons themselves have been identified as potential sources for obtaining stem cells for 

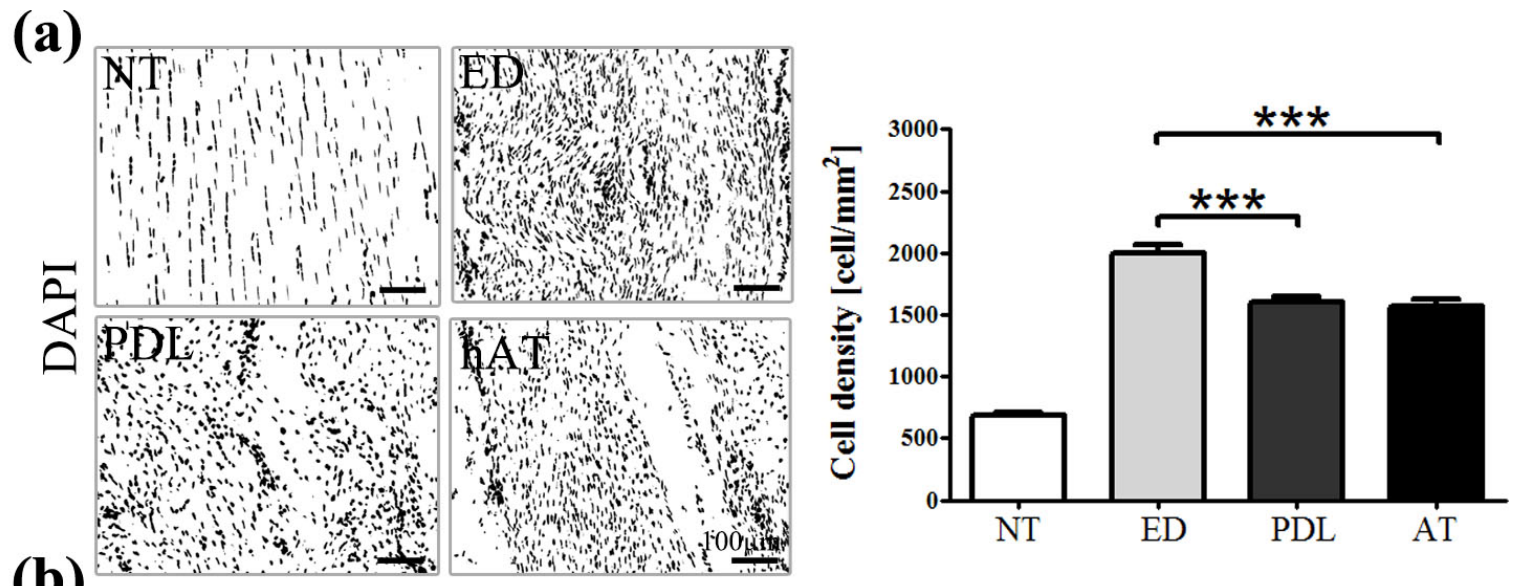

(b)

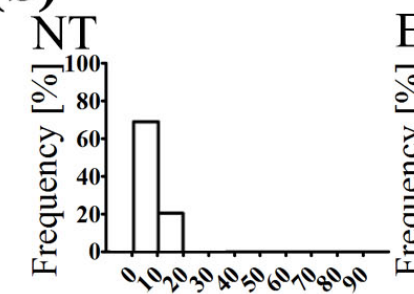

(c)
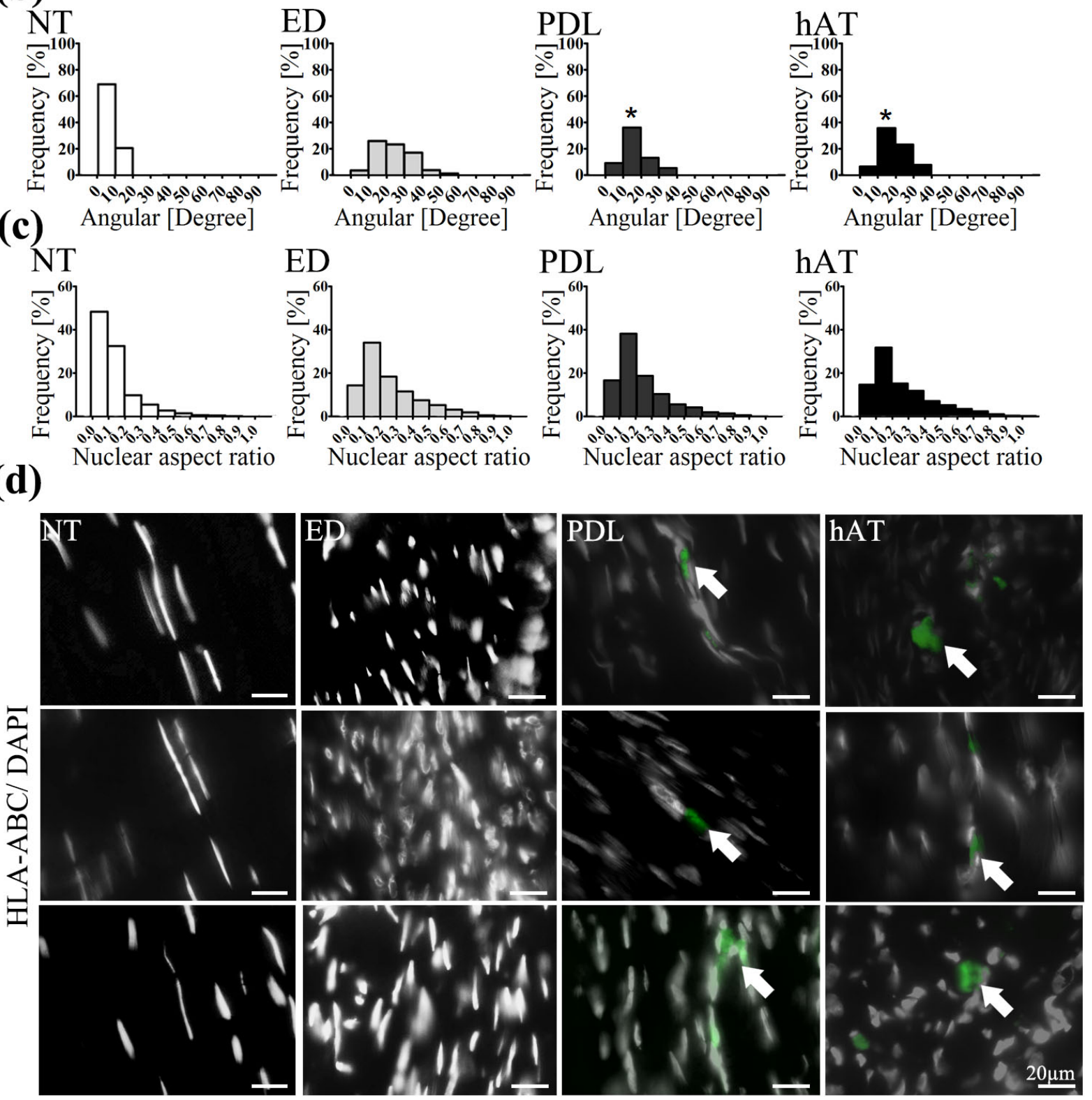

Fig. 5. Analyses of cell density, angular deviation, nuclear aspect ratio and in vivo tracking of the implanted human cells. (a) Representative black and white-converted images of DAPI staining and chart of calculated cell density. Bar charts present mean \pm standard deviation; $* * * p<0.0001$. (b) Frequency distribution of angular deviation (angle between individual nuclear axis and tendon longitudinal axis); ${ }^{*} p=0.01$ for PDL $v s$. ED and ${ }^{*} p=0.02$ for hAT $v s$. ED for the frequency of 10-20 deviation. (c) Frequency distribution of nuclear aspect ratio (NAR, width vs. length of nucleus) in the four study groups, NT, ED, PDL and hAT. (d) Immunostaining for the human-specific antigen HLA-ABC. Representative images for three different animals per group where in positively stained human cells are in green and the nuclear counterstain DAPI in white. 
tendon repair. As mentioned in the introduction, up-to-date, each of these tissues faces several inevitable challenges. With regards to native tendon-derived cells, a clear advantage is that they can differentiate more efficiently directly into tenocytes rather than cells of other lineages. In our previous study (Pietschmann et al., 2013), where we established the clinically relevant full-size Achilles tendon defect model, we compared the healing potential of rat MSC versus rat-derived tenocytes. Based on our histological and biomechanical results demonstrating reduced central ossification, increased tendon-like areas in the site of injury and above all significantly improved ratio of failure strength to tendon cross-section, we strongly concluded that tendon-derived cells hold a clear advantage to MSC. However, one of the most critical issues with the use of tendon cells is the practical limitation behind their obtainment in clinical scenarios. Because of the much easier isolation procedure of periodontal ligament, which can be simply collected from removed teeth during usual orthodontic procedures, as well as the much comparable cellular features of ligamentous and tendinous cells, we hypothesised that PDL could provide a uniquely wellsuited cell source for tendon repair. Hence, in this study we utilised, the previously established by us and evaluated, the healing impact of PDL-derived cell line versus control Achilles tendon-derived cell line (hAT). The main advantages of using cell lines in our experimental model are: being well-characterised, allowing standardisation of the methods and expansion to high cell numbers. However, as discussed below such cell lines are only the first steps in establishing a potent clinical translation protocol because they cannot represent donor variability and be used in patients.

The two study groups, PDL and hAT were compared to non-implanted, but surgically ruptured Achilles tendons (ED group) at 16 weeks postoperatively. This time point corresponded to the late remodelling phase of the tendon healing course wherein major molecular events affecting matrix maturation, cellular organisation and transforming the initial fibrous template to the tissue pattern of healthy tendon are taking place. Based on the combination of tissue and cell morphometric, polarised light microscopic, various histological and immunohistological analyses we found that the PDL group is characterised by smaller tendon diameter, better tissue morphology, advanced matrix maturation and cellular organisation compared to the ED group. This study group was very similar to the control hAT group and both together resembled in several of the studied parameters the NT group. Moreover, in vivo cell tracking demonstrated that the implanted human cells or their progeny can integrate and survive long-term at the side of injury suggesting direct contribution to the healing process. In sum, we can report that PDL-derived cell implantation results in advanced and superior remodelling stage of tendon healing and can be foreseen as a novel alternative to tendon cell source to treat tendon injuries.

The implemented cell lines in our study serve as a great experimental model because they allowed standardised investigation to validate our hypothesis that PDL-derived cells are suitable source for tendon healing. This first step on selecting a high potential cell source in clinically relevant full-size Achilles tendon defect in a small animal model provides the translational bases for designing a true clinical application. The second step should focus on scaling up to comparable tissue dimensions in big animal model such as pig, and using autologous derived PDL cells. Finally, to further speed up the healing, a possible combination of PDL cells with suitable biomaterial carrier should be considered. In such future studies in-depth histological assessment should be combined with key biomechanical measurements and functional performance tests with long-term follow up.

Our novel findings are in line with a recent study by Moshaverinia et al. (2014), who investigated the capacity of two dental tissue-derived cells, periodontal ligament stem cells (PDLSCs) and gingival mesenchymal stem cells (GMSCs) to form tendon-like structures in vivo. The two different cell types were transplanted subcutaneously into mouse dorsal surfaces and the ectopic neo-tendon regeneration was evaluated by qPCR measurements for biglycan, decorin and tenomodulin after 4 weeks and then after 8 weeks by histological and immunohistochemical stainings for tendons-specific protein markers. The authors concluded that PDL as well as GM stem cells can contribute to tendon-like formation in vivo. In this respect, our study presents the next progress step in assessing the healing potential of PDL cells since it demonstrates for the first time that they can indeed enhance the repair of full-size tendon ruptures in a manner that is very comparable to native tendon-derived cells.

Similar to PDL cells, researchers have dealt with other cell types that also have easier accessibility; for example, MSC from human bursa tissue that can be obtained from patients undergoing rotator cuff surgery (Song et al., 2014) and induced pluripotent stem cells (Xu et al., 2013) that can be generated by genetic modifications and banked in high numbers. Moreover, these studies have demonstrated that these cells exhibit a high potential for application in tendon repair. However, at present their use is associated with low efficiency of in vitro expansion; issues with allogenic transplantation; phenotype stability and differentiation control. PDL is a novel attractive cell source because it can be obtained from exfoliated deciduous teeth of children aged 5 onwards and from sound teeth that are normally discarded in frequent routine orthodontic procedures (premolars in class II malocclusion or wisdom teeth removal) in dental clinics. Patient-specific PDL-derived cells can be easily harvested by scratching the surface of the root and in vitro pre-expanded to high numbers in culture with simple standard procedures. Possible bacterial, fungal or mycoplasma contamination can be easily controlled by using antibiotics, antifungal agents or elimination kits. Harvested PDL cells can thereafter be banked up for years ahead and last the phenotype of the cells is very similar in nature to tendon cells, hence no complicated genetic or growth factor manipulations are required. Therefore, the PDL can be considered an ideal source for stem cell banking purposes and be used in therapy of tendon injuries. Several open questions remain to be clarified in following investigations such as the possible combination of PDL cells with suitable biomaterials; the scaling up to dimensions similar to human tendon injuries; the reached 
biomechanical strength; the risk of PDL cell quality loss after long-term storage; the exact technique and time point of application; and the prognosis on periods for patient hospitalisation and post-operative physiotherapy. It will be of great interest, by using adequate animal models, to examine wheatear PDL-derived cells are also suitable for prevention or therapy of tendon/ligament degenerative diseases, that are occurring prior rupture, and for periodontitis. We believe that further research on these questions will be critical to translating our animal model to potential clinical applications.

\section{Conclusions}

In this study, the effect of PDL for cell-based therapy of tendon injuries was investigated using an in vivo model of a full-size rat Achilles tendon defect. Our in-depth results demonstrated that PDL cell-implanted tendons exhibited not only advanced tissue maturation, less ectopic bone formation, more organised collagen fibres, tendon matrix expression corresponding to the final healing stage and better cell-morphometry parameters when compared with the ED group, but were also very similar to the tendons treated with hAT-derived cells. Taken together, our study clearly demonstrates the feasibility of using PDL cells as a novel cell source for tendon repair and strongly recommends this cell type for the future development of innovative regenerative applications for treatment of different tendon or ligament pathologies.

\section{Acknowledgements}

D.D. acknowledged the financial support of the AO Research Fund of the AO Foundation (Project: S-10-74D). C.F.H. was supported by DAAD PhD stipendship program. The authors thank Dr C. Schwarz and Dr J. Kohler for experimental help.

\section{References}

Bi Y, Ehirchiou D, Kilts TM, Inkson CA, Embree MC, Sonoyama W, Li L, Leet AI, Seo BM, Zhang L, Shi S, Young MF (2007) Identification of tendon stem/progenitor cells and the role of the extracellular matrix in their niche. Nat Med 13: 1219-1227.

Bluteau G, Luder HU, De Bari C, Mitsiadis TA (2008) Stem cells for tooth engineering. Eur Cell Mater 16: 1-9.

Docheva D, Muller SA, Majewski M, Evans CH (2015) Biologics for tendon repair. Adv Drug Deliv Rev 84: 222 239.

Docheva D, Padula D, Popov C, Weishaupt P, Pragert M, Miosge N, Hickel R, Bocker W, Clausen-Schaumann H, Schieker M (2010) Establishment of immortalised periodontal ligament progenitor cell line and its behavioural analysis on smooth and rough titanium surface. Eur Cell Mater 19: 228-241.

Hakki SS, Kayis SA, Hakki EE, Bozkurt SB, Duruksu G, Unal ZS, Turac G, Karaoz E (2015) Comparison of mesenchymal stem cells isolated from pulp and periodontal ligament. J Periodontol 86: 283-291.

Hsieh CF, Alberton P, Loffredo-Verde E, Volkmer E, Pietschmann M, Müller P, Schieker M, Docheva D (2016) Scaffold-free Scleraxis-programmed tendon progenitors aid in significantly enhanced repair of full-size Achilles tendon rupture. Nanomedicine, 11:1153-1167.

Ivanovski S, Gronthos S, Shi S, Bartold PM (2006) Stem cells in the periodontal ligament. Oral Dis 12: 358363.

Juneja SC, Veillette C (2013) Defects in tendon, ligament, and enthesis in response to genetic alterations in key proteoglycans and glycoproteins: a review. Arthritis 2013: 154812 .

Kohler J, Popov C, Klotz B, Alberton P, Prall WC, Haasters F, Muller-Deubert S, Ebert R, Klein-Hitpass L, Jakob F, Schieker M, Docheva D (2013) Uncovering the cellular and molecular changes in tendon stem/progenitor cells attributed to tendon aging and degeneration. Aging Cell 12: 988-999.

Lei M, Li K, Li B, Gao LN, Chen FM, Jin Y (2014) Mesenchymal stem cell characteristics of dental pulp and periodontal ligament stem cells after in vivo transplantation. Biomaterials 35: 6332-6343.

Liu J, Yu F, Sun Y, Jiang B, Zhang W, Yang J, Xu GT, Liang A, Liu S (2015) Concise reviews: Characteristics and potential applications of human dental tissue-derived mesenchymal stem cells. Stem Cells 33: 627-638.

Lui PP, Ng SW (2013) Cell therapy for the treatment of tendinopathy-a systematic review on the pre-clinical and clinical evidence. Semin Arthritis Rheum 42: 651-666.

McCloy RA, Rogers S, Caldon CE, Lorca T, Castro A, Burgess A (2014) Partial inhibition of cdk1 in G 2 phase overrides the SAC and decouples mitotic events. Cell cycle 13:1400-1412.

Miura M, Gronthos S, Zhao M, Lu B, Fisher LW, Robey PG, Shi S (2003) SHED: stem cells from human exfoliated deciduous teeth. Proc Natl Acad Sci USA 100: 5807-5812.

Moshaverinia A, Xu X, Chen C, Ansari S, Zadeh HH, Snead ML, Shi S (2014) Application of stem cells derived from the periodontal ligament or gingival tissue sources for tendon tissue regeneration. Biomaterials 35: 2642-2650.

Oragui E, Sachinis N, Hope N, Khan WS, Adesida A (2012) The use of nanotechnology in tendon regeneration and repair. J Stem Cells 7: 121-126.

Pietschmann MF, Frankewycz B, Schmitz P, Docheva D, Sievers B, Jansson V, Schieker M, Muller PE (2013) Comparison of tenocytes and mesenchymal stem cells seeded on biodegradable scaffolds in a full-size tendon defect model. J Mater Sci Mater Med 24: 211-220.

Seo BM, Miura M, Gronthos S, Bartold PM, Batouli S, Brahim J, Young M, Robey PG, Wang CY, Shi S (2004) Investigation of multipotent postnatal stem cells from human periodontal ligament. Lancet 364: 149-155.

Sharma P, Maffulli N (2005a) Basic biology of tendon injury and healing. Surgeon 3: 309-316.

Sharma P, Maffulli N (2005b) Tendon injury and tendinopathy: healing and repair. J Bone Joint Surg Am 87: 187-202. 
Siddiqui NA, Wong JM, Khan WS, Hazlerigg A (2010) Stem cells for tendon and ligament tissue engineering and regeneration. J Stem Cells 5: 187-194.

Simonson DC, Elliott AD, Roukis TS (2016) Catastrophic failure of an infected achilles tendon rupture repair managed with combined flexor hallucis longus and peroneus brevis tendon transfer. Clin Podiatr Med Surg 33: $153-162$.

Song N, Armstrong AD, Li F, Ouyang H, Niyibizi C (2014) Multipotent mesenchymal stem cells from human subacromial bursa: potential for cell based tendon tissue engineering. Tissue Eng Part A 20: 239-249.

Xu W, Wang Y, Liu E, Sun Y, Luo Z, Xu Z, Liu W, Zhong L, Lv Y, Wang A, Tang Z, Li S, Yang L (2013) Human iPSC-derived neural crest stem cells promote tendon repair in a rat patellar tendon window defect model. Tissue Eng Part A 19: 2439-2451.

\section{Discussion with Reviewer}

Christopher Niyibizi: For future clinical application, PDL cells would be harvested from different individuals, is there a worry for potential immune response to the donor cells by the recipients?

Authors: We foresee the use of PDL cells in two applicative modes: autologous or allogeneic. The autologous PDL cell implantation eliminates problems with immune rejection of the grafted material. In cases of low harvest of PDL cells allogeneic PDL cell implantation comes in question.
Interestingly, two studies have already demonstrated that PDL-derived stem/progenitor cells possess low immunogenicity and actually can play immunomodulatory roles. Ding et al. (2010), observed no immune rejection of allogenic PDL cells transplanted in a swine model of periodontitis. Kim et al. (2010) showed that human and canine PDL cells have profound immunomodulatory effect (inhibition of cell division) on human peripheral blood mononuclear cells. The above results suggest that PDL donor cells can circumvent immune rejection; however, follow up xenogenic in vivo studies are necessary to strengthen such findings prior clinical testing.

\section{Additional References}

Ding G, Liu Y, Wang W, Wei F, Liu D, Fan Z, An Y, Zhang C, Wang S (2010) Allogeneic periodontal ligament stem cell therapy for periodontitis in swine. Stem Cell, 28: 1829-1838.

Kim HS, Kim KH, Kim SH, Kim YS, Koo KT, Kim TI, Seol YJ, Ku Y, Rhyu IC, Chung CP, Lee YM (2010) Immunomodulatory effect of canine periodontal ligament stem cells on allogenic and xenogenic peripheral blood mononuclear cells. J Periodontal Implant Sci 40: 265-270

Editor's note: The Scientific Editor responsible for this paper was Juerg Gasser. 